\title{
Nanoscale Surface Redox Chemistry Triggered by Plasmon- Generated Hot Carriers
}

\section{Journal Article}

Author(s):

Yin, Hao; Lan, Jing-Gang; Goubert, Guillaume (D); Wang, Yao-Hui; Li, Jian-Feng; Zenobi, Renato (1)

Publication date:

2019-11

Permanent link:

https://doi.org/10.3929/ethz-b-000369629

Rights / license:

In Copyright - Non-Commercial Use Permitted

Originally published in:

Small 15(47), https://doi.org/10.1002/smll.201903674 


\section{small}

Postfach 101161 69451 Weinheim

Germany

Courier services:

Boschstraße 12

69469 Weinheim

Germany

Tel.: (+49) 6201606235

Fax: (+49) 6201606500

E-mail: small@wiley.com

\section{WILEY-VCH}

Dear Author,

Please correct your galley proofs carefully and return them no more than four days after the page proofs have been received.

Please limit corrections to errors already in the text; cost incurred for any further changes or additions will be charged to the author, unless such changes have been agreed upon by the editor.

The editors reserve the right to publish your article without your corrections if the proofs do not arrive in time.

Note that the author is liable for damages arising from incorrect statements, including misprints.

Please note any queries that require your attention. These are indicated with a $Q$ in the PDF and a question at the end of the document.

Reprints may be ordered by filling out the accompanying form.

Return the reprint order form by fax or by e-mail with the corrected proofs, to WileyVCH : small@wiley.com
To avoid commonly occurring errors, please ensure that the following important items are correct in your proofs (please note that once your article is published online, no further corrections can be made):

- Names of all authors present and spelled correctly

- Titles of authors correct (Prof. or Dr. only: please note, Prof. Dr. is not used in the journals)

- Addresses and postcodes correct

- E-mail address of corresponding author correct (current email address)

- Funding bodies included and grant numbers accurate

- Title of article OK

- All figures included

- Equations correct (symbols and sub/superscripts)

Corrections should be made directly in the PDF file using the PDF annotation tools. If you have questions about this, please contact the editorial office. The corrected PDF and any accompanying files should be uploaded to the journal's Editorial Manager site. 


\section{Author Query Form}

Journal

SMLL

Article

smll201903674

Dear Author,

During the copyediting of your manuscript the following queries arose.

Please refer to the query reference callout numbers in the page proofs and respond to each by marking the necessary comments using the PDF annotation tools.

Please remember illegible or unclear comments and corrections may delay publication.

Many thanks for your assistance.

\begin{tabular}{|c|l|c|}
\hline Query No. & \multicolumn{1}{|c|}{ Description } & Remarks \\
\hline Q1 & $\begin{array}{l}\text { Please confirm that forenames/given names (blue) and surnames/family names (vermilion) have been } \\
\text { identified correctly. }\end{array}$ & \\
\hline Q2 & Please provide the highest academic title (either Dr. or Prof.) for all authors, where applicable. & $\begin{array}{l}\text { Open access publication of this work is possible via Wiley OnlineOpen. Information about OnlineOpen, the } \\
\text { article publication charges (APCs), and Wiley's agreements with various institutions to cover the APCs for } \\
\text { their affiliated authors can be found at: https://authorservices.wiley.com/author-resources/Journal-Authors/ } \\
\text { licensing-open-access/open-access/onlineopen.html. } \\
\text { Q3 }\end{array}$ \\
\hline $\begin{array}{l}\text { The cost of publishing your manuscript OnlineOpen may be covered by one of Wiley's national agreements. } \\
\text { wiley.com/author-resources/Journal-Authors/open-access/affiliation-policies-payments/index.html. } \\
\text { Eligibility for these funds is based on the affiliation of the primary corresponding author. }\end{array}$ & $\begin{array}{l}\text { Refs. [17] and [39] and [34] and [38] were identical, so we have deleted the later and renumbered the remaining. } \\
\text { Please validate. }\end{array}$ \\
\hline
\end{tabular}

Please confirm that Funding Information has been identified correctly.

Please confirm that the funding sponsor list below was correctly extracted from your article: that it includes all funders and that the text has been matched to the correct FundRef Registry organization names. If a name was not found in the FundRef registry, it may not be the canonical name form, it may be a program name rather than an organization name, or it may be an organization not yet included in FundRef Registry. If you know of another name form or a parent organization name for a "not found" item on this list below, please share that information.

\begin{tabular}{|l|l|}
\hline FundRef Name & FundRef Organization Name \\
\hline Sino-Swiss Science and Technology Cooperation & \\
\hline NSFC & \\
\hline Sino-Swiss Science and Technology Cooperation & \\
\hline
\end{tabular}




\section{FULL PAPERS}

H. Yin, J.-G. Lan, G. Goubert,

Y.-H. Wang, J.-F. Li,*'

R. Zenobi*

1903674

Nanoscale Surface Redox Chemistry

Triggered by Plasmon-Generated Hot

Carriers

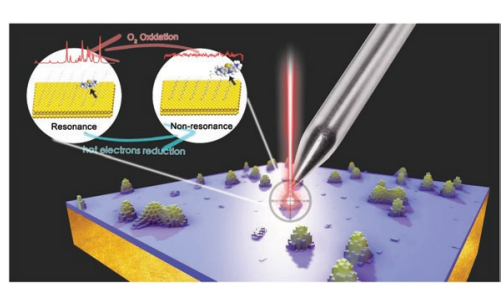

Tip-enhanced Raman intensity fluctuations of surface bound resonant dyes molecules reveal plasmonic hot electron-mediated redox reactions, which switch between the reduced/oxidized molecular forms. The hot electrons transfer mechanism through a thiol spacer layer is discussed in the light of ambient tip-enhanced Raman spectroscopy and surface-enhanced Raman spectroscopy investigations including spacer-layer and applied electric field dependence analyses. 


\section{Nanoscale Surface Redox Chemistry Triggered by Plasmon-Generated Hot Carriers}

Direct photoexcitation of charges at a plasmonic metal hotspot produces energetic carriers that are capable of performing photocatalysis in the visible. However, the mechanisms of generation and transport of hot carriers are still not fully understood and under intense investigation because of their potential technological importance. Here, spectroscopic evidence proves that the reduction of dye molecules tethered to a $\mathrm{Au}(111)$ surface can be triggered by plasmonic carriers via a tunneling mechanism, which results in anomalous Raman intensity fluctuations. Tip-enhanced Raman spectroscopy (TERS) helps to correlate Raman intensity fluctuations with temperature and with properties of the molecular spacer. In combination with electrochemical surface-enhanced Raman spectroscopy, TERS results show that plasmoninduced energetic carriers can directly tunnel to the dye through the spacer. This organic spacer chemically isolates the adsorbate from the metal but does not block photo-induced redox reactions, which offers new possibilities for optimizing plasmon-induced photocatalytic systems.

\section{Introduction}

Photocatalysis in the visible with plasmon-induced energetic carriers (so-called hot electrons) has received significant attention. ${ }^{[1]}$ Hot electrons enable a wide range of applications, e.g., water splitting, ${ }^{[2]}$ ethylene epoxidation, ${ }^{[3]}$ and hydrogen dissociation. ${ }^{[4]}$ However, the short lifetimes of hot carriers (less than picoseconds) greatly reduces the quantum efficiency of plasmon-mediated reactions, which requires separating charge

H. Yin, Y.-H. Wang, Prof. J.-F. Li, Prof. R. Zenobi

MOE Key Laboratory of Spectrochemical Analysis and Instrumentation

State Key Laboratory of Physical Chemistry of Solid Surfaces

iChEM

College of Chemistry and Chemical Engineering

Xiamen University

Xiamen 361005, China

E-mail: zenobi@org.chem.ethz.ch; li@xmu.edu.cn

H. Yin, Dr. G. Goubert, Prof. R. Zenobi

Department of Chemistry and Applied Biosciences

ETH Zurich

CH-8093 Zurich, Switzerland

J.-G. Lan

Department of Chemistry

University of Zurich

$\mathrm{CH}-8057$ Zurich, Switzerland

The ORCID identification number(s) for the author(s) of this article can be found under https://doi.org/10.1002/smll.201903674.

DOI: $10.1002 / \mathrm{smll} .201903674$ carriers followed by transfer to the reacting 11 molecules. Surface-enhanced and tip- 12 enhanced Raman spectroscopy (SERS 13 and TERS) are ideal analytical tools to 14 elucidate the mechanism of plasmon- 15 mediated reactions at the molecular level, 16 since they provide chemical information 17 with high sensitivity ${ }^{[5]}$ Focusing on surface 18 molecular Raman spectroscopy, several 19 groups have reported plasmon-mediated 20 surface reactions, especially the coupling 21 reaction of p-aminothiophenol (pATP). ${ }^{[6,7]} 22$ However, because reactants are usually 23 directly absorbed on the metal surface, 24 the substrate could also catalyze the reac- 25 tion, via different pathways. For example, 26 in the presence of $\mathrm{O}_{2}$, the widely studied 27 coupling reaction of pATP on silver is ther- 28 modynamically favored and most likely 29 triggered by adsorbed oxygen rather than 30 by hot electrons. ${ }^{[8,9]}$ One way to minimize 31 the effect of the metal-molecule interaction is by introducing a 32 dielectric spacer layer between the metal and the adsorbate. $\quad 33$

The high sensitivity of SERS is mostly due to the local elec- 34 tromagnetic field enhancement in "hotspots" (nanoscale regions 35 where hot electrons primarily appear ${ }^{10]}$ ). Unfortunately, the 36 enhancement in the randomly distributed hotspots on an SERS 37 substrate can vary enormously. On the contrary, in TERS, a 38 single metallic tip apex is used as a hotspot and is held in close 39 proximity to the sample using a scanning probe microscope..$^{[11,12]} \quad 40$ Charge carrier generation is thus limited to a single hotspot in 41 TERS, which renders it a better spectroscopic tool for studying 42 plasmon-mediated reactions. Here, we report how hot carriers 43 generated under a plasmonic TERS tip can trigger reduction of 44 Nile blue (NB) and methylene blue (MB) covalently tethered to a 45 $\mathrm{Au}(111)$ surface via spacers of different length. The dyes NB and 46 MB are used as a prototype reversible redox chromophores to 47 study the generation and transfer of hot carriers, and the effect 48 of temperature and spacer length. We studied the TERS signal 49 intensity fluctuations to understand the transport mechanism of 50 hot carriers through the spacer to the dye molecules. 51

\section{Results and Discussion}

2.1. Chemically Bonded Dye Molecules on Au(111) Surface 56

The protonation of dye molecules such as MB and NB results 58 in the formation of the corresponding leuco-dye, which have 59 


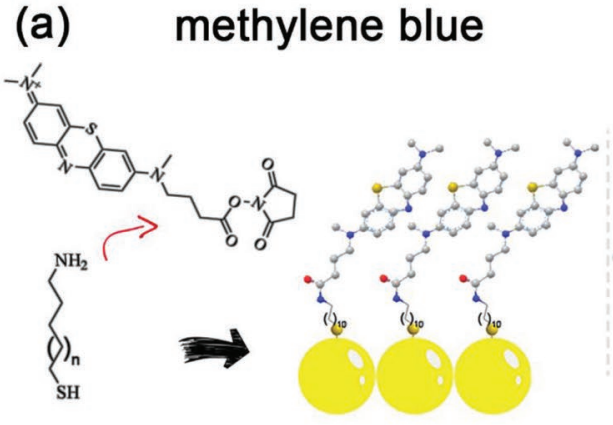

$\mathrm{Au}(111)$

(b)

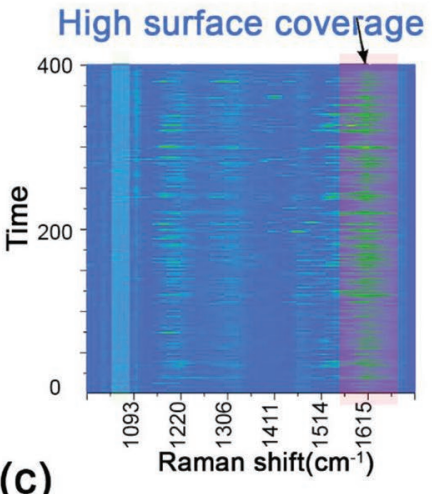

(c)

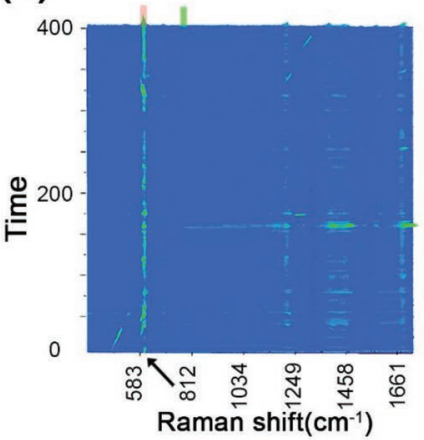

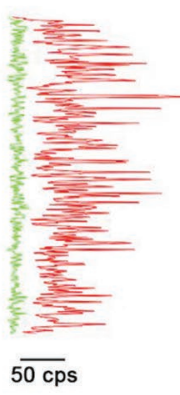

Low surface coverage
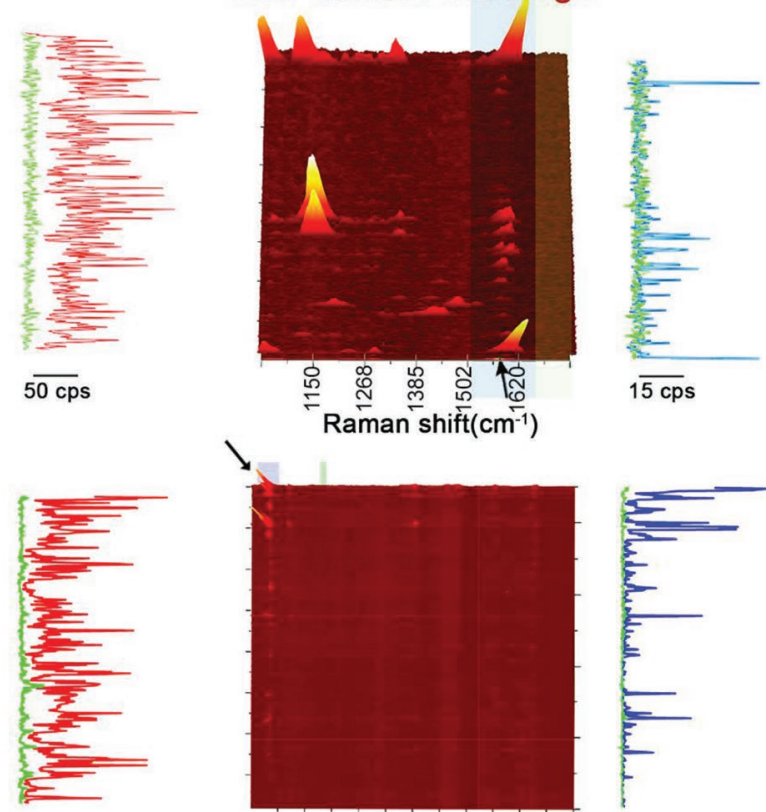

$\widetilde{50 \mathrm{cps}}$

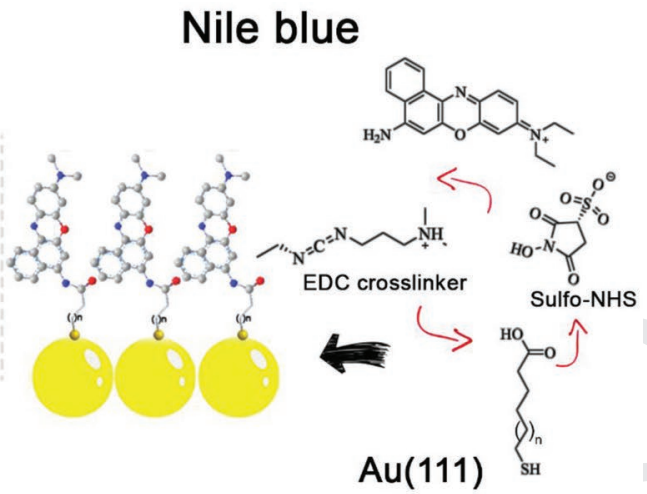

1

2

3

4

5

Figure 1. a) Carbodiimide crosslinking methods used for surface molecular modification: MB (left)/NB (right). Intensity fluctuation of b) MB 42 $\left(\approx 1615 \mathrm{~cm}^{-1}\right)$ and c) NB $\left(\approx 598 \mathrm{~cm}^{-1}\right)$ during TERS measurements at different surface coverages: high coverage (left) and low coverage (right). The green lines correspond to the background noise.

distinctive features in their respective UV-vis absorption spectra. ${ }^{[13]}$ We used carbodiimide crosslinker chemicals to bind the dyes to the end of an alkanethiol self-assembled monolayer (SAM; Figure 1a). Upon binding to the surface, their molecular orbitals are mostly unperturbed by the metallic $\mathrm{Au}(111)$ substrate because of the insulating effect of the self-assembled thiol monolayer. The cyclic voltammograms of the covalently tethered dyes exhibit two distinct peaks, rather than one peak for physisorbed molecules ${ }^{[14]}$ (Figure S1, Supporting Information). We also adjusted the density of surface functional groups in order to control the dye coverage by using a mixed thiolate SAM. ${ }^{[15]}$ We chose two surface coverages: 1) full coverage of the Au surface by a single reactive thiolate SAM terminated with amino/carboxyl group (called high surface coverage below), and
2) deposition of a mixed SAM with a 1:500 ratio $\left(20 \times 10^{-6}: 10 \times\right.$ $10^{-3} \mathrm{M}$ ) of reactive and nonreactive thiolates (low surface coverage). We used thiol (see the Experimental Section) to form a compact SAM layer. ${ }^{[16]}$ Surface coverage measurements indicated that the number of dye molecules in the hotspot was around 1500-2000 at high coverage and 10-20 at low coverage (Figures S1 and S2 and Equation (S1), Supporting Information).

\subsection{Intensity Fluctuations in TERS}

In our system, a silver tip is used as a scanning tunneling 57 microscopy (STM) probe and as an optical antenna, and 58 $\mathrm{Au}(111)$ as a substrate, forming a gap mode plasmon with 59 
stronger field enhancement. One of our main observables was Raman intensities for MB (at $1615 \mathrm{~cm}^{-1}$, Figure $1 \mathrm{~b}$ ) and NB (at $598 \mathrm{~cm}^{-1}$, Figure 1c) in single point TER measurements at different surface coverages. These intensities exhibited large fluctuations, which we interpret to be due to transient reduction of dye molecules. To corroborate this interpretation, we compared this data against that from a series of common molecules with similar Raman cross-sections, but which were not affected by redox chemistry. We included nonresonant molecules and one molecule with an electronic resonance, malachite green isothiocyanate (MGITC). ${ }^{[17]}$ Adsorbed MGITC on $\mathrm{Au}$ is less prone to undergo a redox reaction, as described by Nie and co-workers:[18] MGITC adsorbs via two different molecular moieties, the dimethylamino and isothiocyanate groups, which irreversibly locks the adsorbed molecule in its delocalized electronic structure. Upon adsorption, the central carbon atom can no longer change from $\mathrm{sp}^{2}$ to $\mathrm{sp}^{3}$ hybridization, which means that reduction of MGITC is inhibited. In all controls, a much more even signal intensity was found (Figure S3, Supporting Information), irrespective of the molecule's chemical structure or of the presence of an electronic resonance.

Upon reduction, the molecular electronic resonance is lost for both NB and MB, and the corresponding TERS amplitude is greatly decreased. The signal recovers spontaneously, which we interpret as reoxidation of the leuco-dyes in the presence of $\mathrm{O}_{2}$ in the ambient. We propose that the interplay between plasmon-mediated reduction and spontaneous oxidation in air results in the large intensity fluctuations dominated in the TERS signal. We acquired TER spectra at low coverage to statistically analyze the proportion of the oxidized and reduced forms, where the intensity fluctuations exhibited a blinkinglike (on/off) behavior. Furthermore, compared to ambient conditions, spectra characteristic of the resonant form of the dye appeared more frequently when we measured in an $\mathrm{O}_{2}$ enriched atmosphere as displayed in Figure 1b,c and Figure S4 in the Supporting Information. There is a large TER intensity difference between the on and off states: when in the on state (dyes in the oxidized form) the dye fingerprint spectrum is visible; when in the off state (dyes in the reduced form) only noise is obtained because of the hundredfold weaker signal of the leuco-dye.

Blinking is also a typical phenomenon in single-molecule spectroscopy. ${ }^{[19 a]}$ However, the mean on- and off-times would be on the millisecond timescale for chemically tethered $\mathrm{MB}$, according to Orrit's single-molecule fluorescence research. ${ }^{\text {[19b] }}$ These researchers used a step-detection algorithm to extract the on- and off-times of a single chemically bonded MB molecules, which were found to follow an exponential distribution with mean on- and off-times of $10.0 \pm 0.7$ and $39 \pm 2 \mathrm{~ms}$, whereas in this study, the typical acquisition time per spectrum was $5 \mathrm{~s}$. This long averaging time precludes detection of the much shorter blinking typical of single-molecule Raman experiments and averages out small fluctuations in vibrational or rotational changes in a molecule's local environment. Additionally, we expect to probe 10-20 molecules at any given time in the hotspot. We can thus safely rule out the possibility that the intensity fluctuations observed here are due to single-molecule behavior. Thermal drift of the STM tip relative to the sample is also inevitable in ambient conditions.
Drift could also cause intensity fluctuations, e.g., surface 1 domains would cause the number of molecules within the 2 hotspot to greatly change with pattern. To demonstrate that a 3 uniform surface without nano-domains was formed, we used 4 a novel probe molecule, aminomethyl benzonitrile (AMBT). 5 AMBT was also attached to the surface using the 1-ethyl-3-(3- 6 dimethylaminopropyl)-carbodiimide hydrochloride (EDC)/N- 7 hydroxysulfosuccinimide (NHS) procedure (Figure S5, Sup- 8 porting Information), but cannot participate in any redox 9 reaction. Compared to the NB intensity at $598 \mathrm{~cm}^{-1}$ that fluctu- 10 ated at low surface coverage, the TERS intensity at 1611 and 11 $2237 \mathrm{~cm}^{-1}$ in the spectrum of AMBT was relatively stable, indi- 12 cating the existence of a uniform substrate (Figure 2a,b). There- 13 fore, we can conclude that the signal fluctuations did not result 14 from thermal drift over a heterogenous surface. 15

It is challenging to obtain spectra with a high enough signal- 16 to-noise ratio of both nonresonant leuco-dyes by TERS. We 17 turned to SERS, which can provide a higher signal at similar 18 conditions, to further confirm that a redox reaction takes place. 19 As can be seen in Figure 2c, most observed Raman bands origi- 20 nate from the oxidized form of MB (Raman resonant). These 21 modes were assigned with the help of qualitative density func- 22 tional theory (DFT) calculations, using a relatively small basis 23 set. Under continuous irradiation by the $633 \mathrm{~nm}$ laser, the 24 intensity of the Raman peaks at 1213 and $1603 \mathrm{~cm}^{-1}$ increased, 25 both stem from the reduced form of MB (leuco-MB, Figure S13, 26 Supporting Information), while the bands of the nonreduced 27 form of MB decreased. After stopping the laser illumination, 28 the intensities of the 1213 and $1603 \mathrm{~cm}^{-1}$ bands decreased 29 again, while bands characteristic of the oxidized dye recovered. 30 Very likely, in the case of SERS, this is again the result of leuco- 31 dye oxidation by $\mathrm{O}_{2}$ in the air.

\subsection{Hot Electron Transfer Mechanism}

In our metal-SAM-dye system, carriers must pass through the 37 thiolate SAM to trigger reduction of the dye. In other systems 38 and experiments, charge separation and transfer were found to 39 proceed on a fast timescale, from picoseconds ${ }^{[20]}$ to microsec- 40 onds. ${ }^{[21]}$ The efficiency of the charge transfer strongly affects 41 the overall quantum yield. ${ }^{[22-24]}$ Aiming for further confirma- 42 tion that hot electrons possess the same transfer properties as 43 lower energy electrons, ${ }^{[25,26]}$ we considered two potential charge 44 transfer mechanisms: incoherent, thermally activated hop- 45 ping, or coherent, nonresonant tunneling (Figure 3). The two 46 processes have been widely discussed in the molecular elec- 47 tronics field, ${ }^{[27]}$ suggesting that the tunneling is more distance- 48 sensitive, while hopping is more temperature-sensitive. ${ }^{[28]} \quad 49$

We investigated the hot carrier transport mechanism by com- 50 paring the occurrence of the on/off states as a function of dye- 51 substrate distance and temperature. Since the distance from 52 the molecular dipole of the dye to the substrate is roughly the 53 SAM thickness, we selected two thiols with different lengths 54 $(\approx 0.5 \mathrm{~nm}$ for a 5 -carbon-long chain and $\approx 1 \mathrm{~nm}$ for a 11-carbon- 55 long chain). Figure $4 \mathrm{a}, \mathrm{b}$ presents the electronic structures of 56 chemically tethered MB and NB: the metal substrate has no sig- 57 nificant influence on the lowest unoccupied molecular orbital 58 (LUMO). To confirm that changes in the thiolate length do not 59 
(a)

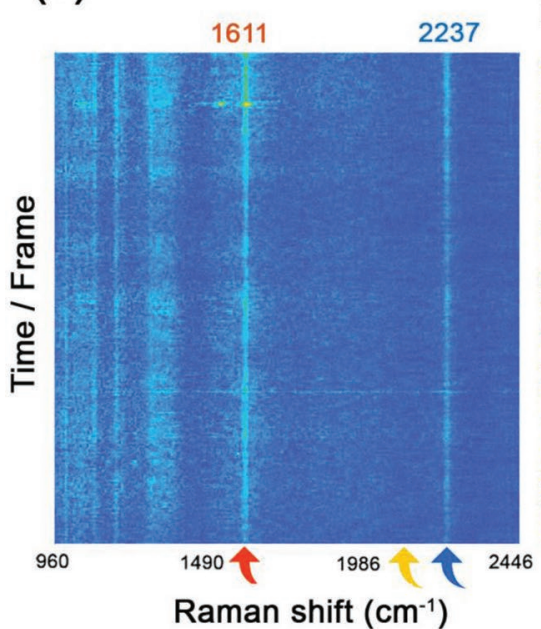

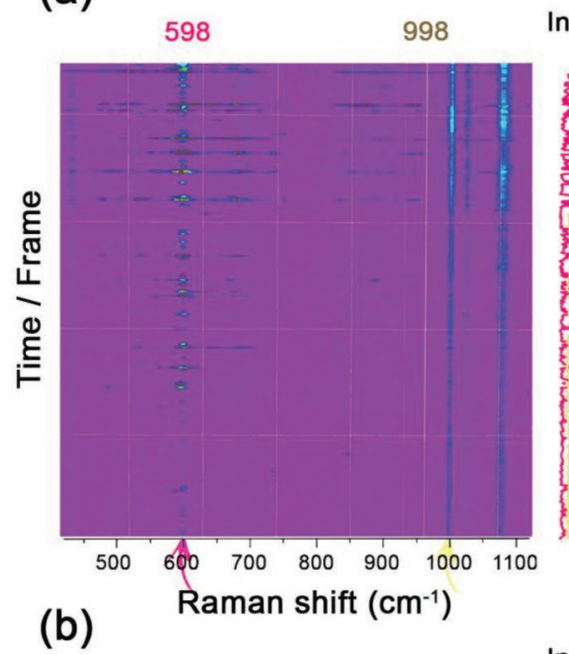
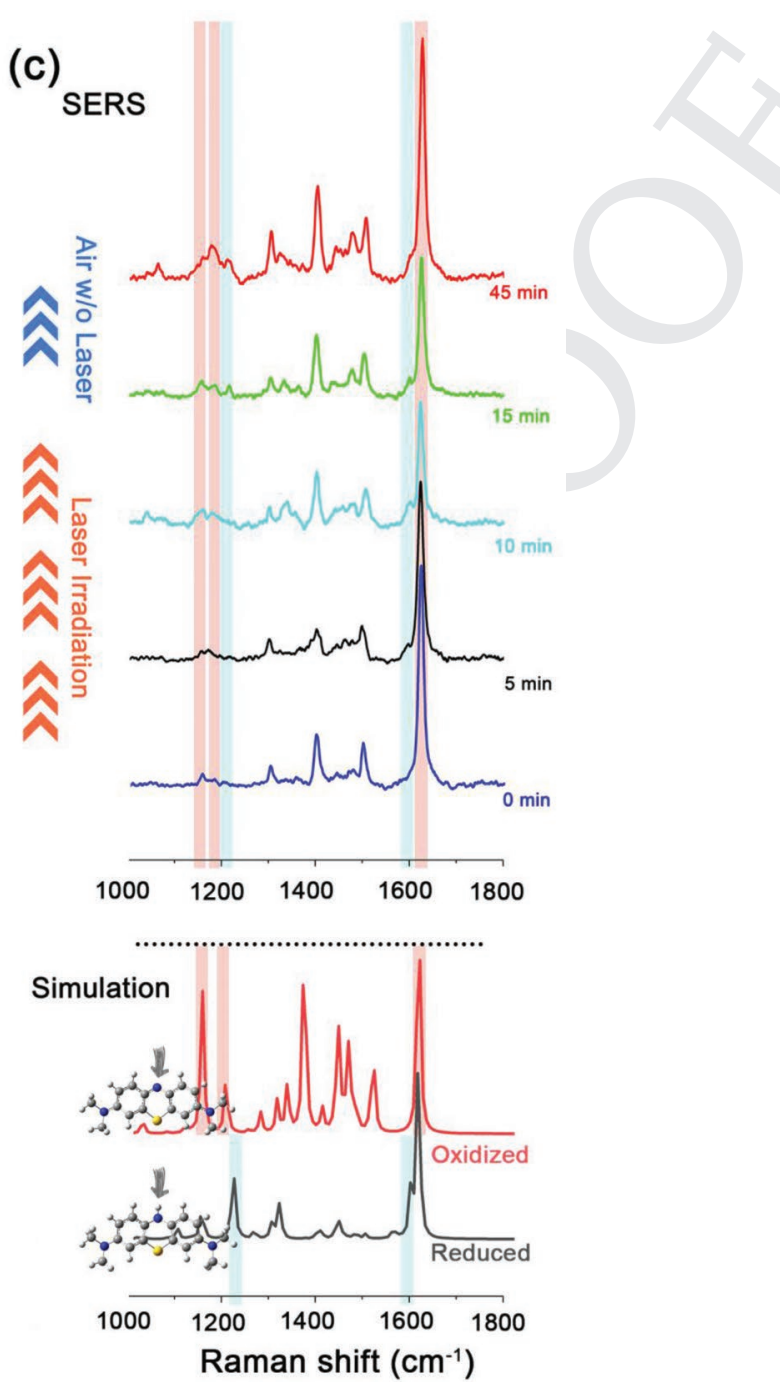

1 2 3 4 5 6 7 8

Figure 2. a) TERS intensity fluctuations for NB bound to mercaptobenzoic acid/thiophenol at low surface coverage. b) TERS intensity fluctuations 37 for (aminomethyl)benzonitrile bound to mercaptododecanoic acid/undecanethiol via the same procedure and at the same coverage. Measurement conditions: $633 \mathrm{~nm}, 0.03 \mathrm{~mW}$. c) SERS experiment with MB after $633 \mathrm{~nm}$ laser illumination at various times, and corresponding simulation of the Raman spectra of the reduced/oxidized form.
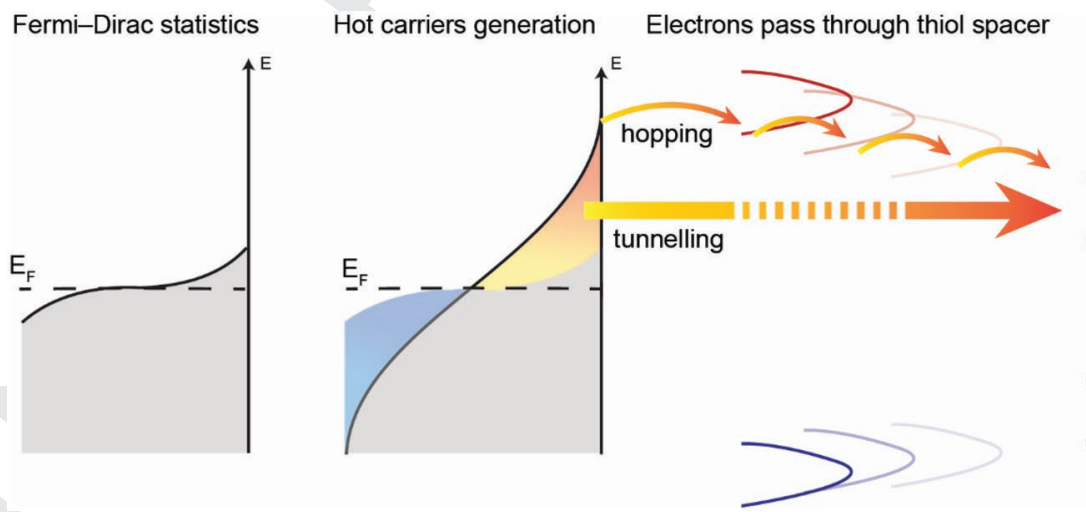

Dyes orbitals

Figure 3. Potential mechanisms for generation of hot electrons and transfer to molecule orbitals. After plasmon excitation, the Landau damping and electron-electron interaction will form hot electrons that will pass through the spacers to molecular orbitals either by a tunneling or a hopping mechanism. 
(a)

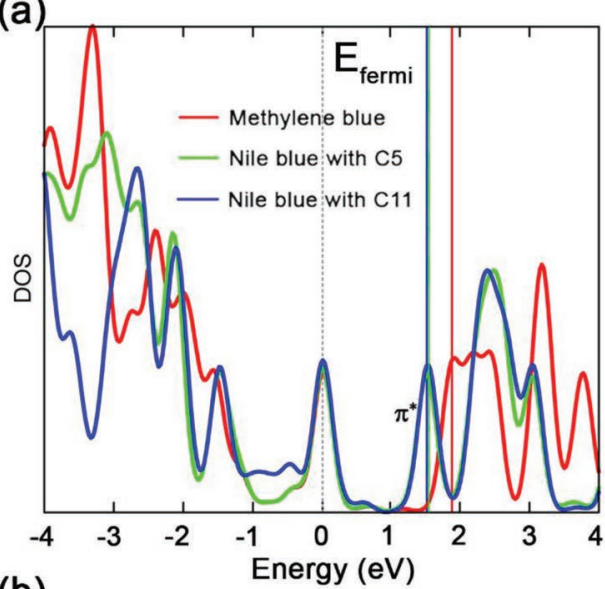

(b)

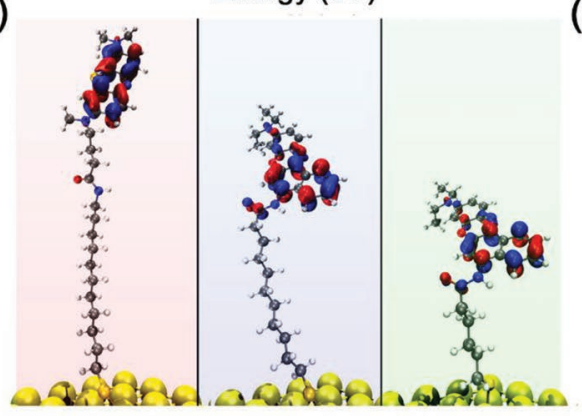

(c)

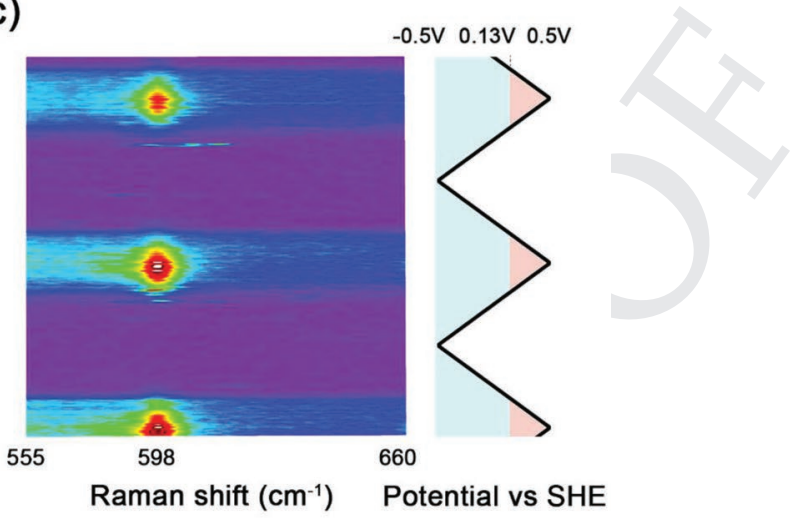

(d)

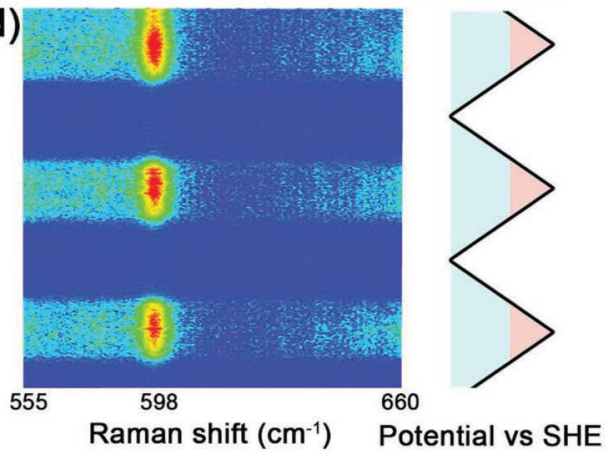

1

2

3

4

5

6

7

8

Figure 4. PDOS of the molecules adsorbed on the a) $\mathrm{Au}(111)$ surface and b) the corresponding isosurface of the LUMO wavefunction. EC-SERS 29 measurements of chemically tethered NB molecules in $0.1 \mathrm{~mol} \mathrm{~L}^{-1} \mathrm{HClO}_{4}$ with the c) $\mathrm{C} 5 /$ d) $\mathrm{C} 11$ thiolate SAM spacers.

affect the thermodynamics of the redox reaction, ${ }^{[29]}$ we measured the chemically tethered NB electrochemical (EC)-SERS signals with different thiolate spacers (Figure 4c,d). EC-SERS measurements showed a significant but reversible Raman intensity change with the potential for several cycles. The reversible redox reaction onset appeared at a similar potential, irrespective of the thiolate layer thickness. ${ }^{[30]}$

We analyzed the ratio of the oxidized to the reduced form at low surface coverage. During TERS experiments, this ratio is more than doubled, from $21 \%$ to $47 \%$, when we chose the $1 \mathrm{~nm}$ thiolate spacer instead of the $0.5 \mathrm{~nm}$ thiolate spacer, as seen in Figure 5a. This change indicates that the reversible redox reaction is greatly affected by the distance between the dye and the metal surface. We then selected a longer thiolate spacer with a 15-carbon chain, surprisingly, the anomalous intensity fluctuations were not observed (Figure S6, Supporting Information) and the redox reaction did not happen as expected. We also observed a clear decrease of the Raman signal intensity and the NB fluorescence background, which was quenched with shorter spacers as shown in Figure S7 in the Supporting Information. This indicates that the metaldye interaction was weakened and the hot electrons cannot trigger the reaction with such a long distance between the dyes and the metallic substrate. This also excludes other longdistance (more than $10 \mathrm{~nm}$ ) energy transfer processes such as plasmon-coupled resonance energy transfer. ${ }^{[31 a, b]}$ We also considered a plasmonic charge transfer/tunneling mechanism which could trigger a vibrational Stark effect (as observed, e.g., for $\mathrm{CO},{ }^{[32 a]}$ nitrile, ${ }^{[32 b]}$ cyanide $^{[33 c]}$. We selected ferent thiolate linkers $(\mathrm{C} 5 / \mathrm{C} 11)$ to probe the nitrile band shift of AMBT. Almost no band shift was observed (Figure S5.2, 34 Supporting Information), which indicates that this mechanism 35 is of minor importance in our system. To further exclude the 36 possibility that the Ag tip generates the hot electrons for the 37 redox reaction, we developed a wet coating method, ${ }^{[3]}$ to prepare 38 insulating silica shell-isolated tips (Figure S8, Supporting Infor- 39 mation). The intensity fluctuations observed when using these 40 shell-isolated tips are similar to those of bare tips (Figure S9, 41 Supporting Information), which indicates that the hot electrons 42 mainly originate from the Au substrate rather than from the 43 $\mathrm{Ag}$ tip. This distance dependence is consistent with a tunneling 44 mechanism where electrons transfer directly through the 45 $<1 \mathrm{~nm}$ thick barrier. The shorter the barrier, the higher the 46 probability for electrons to tunnel through, which in turn leads 47 to a higher rate for the reduction reaction. Since hopping is 48 sensitive to the surrounding temperature, and we carried out 49 an experiment when we maintained the substrate at different 50 temperatures $\left(30,40\right.$, and $50{ }^{\circ} \mathrm{C}$; even higher temperature may 51 lead to the degradation of thiol-Au bonds and surface dye mole- 52 cules $\left.^{[34]}\right)$. It is also worthy to mention that, in agreement with 53 our previous results, ${ }^{[35]}$ laser-induced thermal damage of the 54 sample did not occur under weak laser intensity $(30 \mu \mathrm{W})$. We 55 do not observe any signs of thermal damage from local thermal 56 contribution, ${ }^{[36]}$ such as broad carbon peaks. Figure 5b shows 57 that the oxidized/reduced ratio varies less with temperature 58 (22-26\%) than it does with the distance (21-47\%). Considering 59 


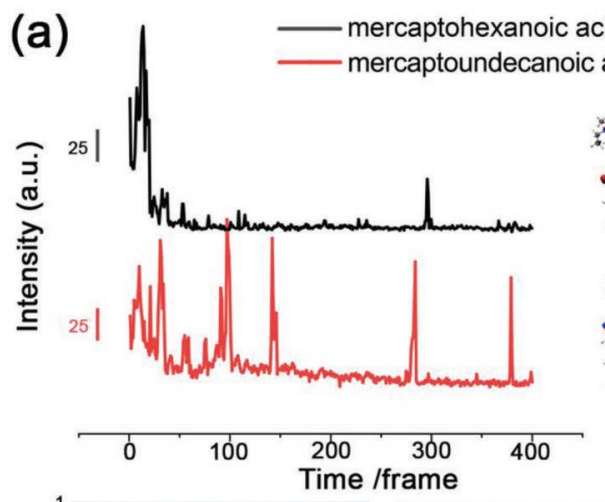

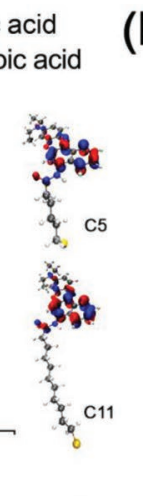

(b)
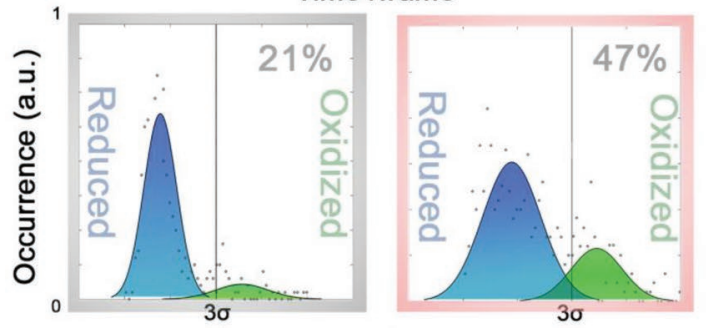
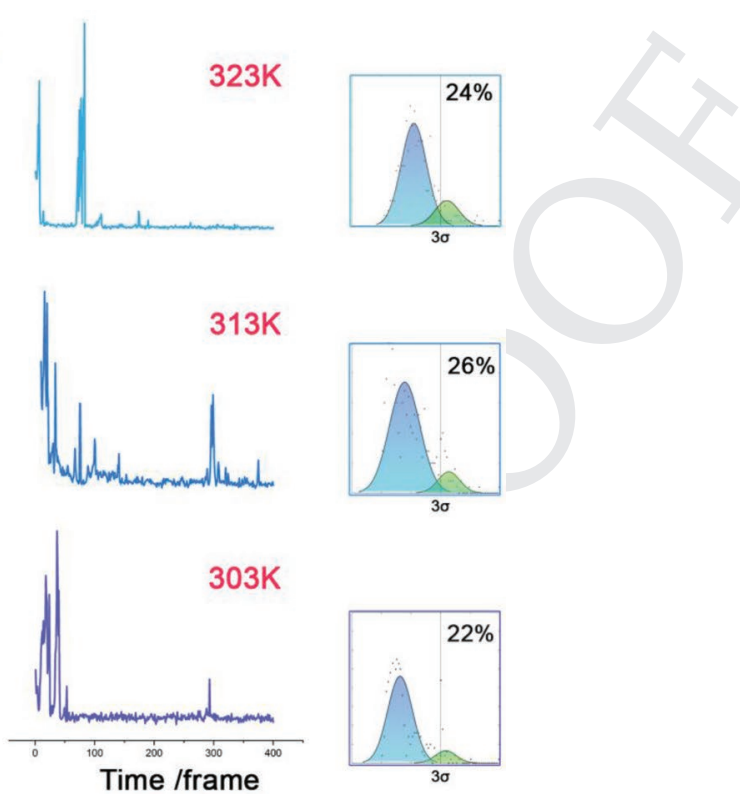

1

2

3

4

5

6

7

8

9

10

11

12

13

14

15

16

17

18

19

20

21

22

Figure 5. a) Intensity fluctuations of the $598 \mathrm{~cm}^{-1}$ NB TERS band and statistical analysis at low surface coverage with different thiolate spacer lengths, 23 and b) intensity fluctuations of the $598 \mathrm{~cm}^{-1}$ NB TERS band and statistical analysis at low surface coverage at different temperatures. A mercaptohexa- 24 noic acid/pentanethiol layer was used in (b).

that this distance was in the sub-nm range and that the saturated alkyl chains have a poor electron affinity, we are assuming that in our dye/SAM/metal system, the charge transfer is better described by a tunneling than by a hopping mechanism which is similar to tunneling of lower energy electrons. ${ }^{[27]}$

\section{Conclusions}

In summary, we observed large intensity fluctuations in the Raman spectra of covalently tethered $\mathrm{MB}$ and NB during ambient TERS measurements, especially at low surface coverage. We found that these fluctuations result from the reduction triggered by plasmon-induced carriers in a single hotspot and spontaneous reoxidation involving ambient oxygen, which results in the reversible appearance and disappearance of the dye's resonant structure. Our study thus extends the range of plasmon-triggered reactions into surface reversible redox reactions. We also demonstrate that the transfer of energetic electrons through the donor-bridge-acceptor structure (dye/SAM/ metal surface) is dominated by tunneling rather than hopping. Properties of short molecular barriers (thickness, conductivity, chemical reactivity) could be used to tune plasmon-mediated reactions. We anticipate that the new degrees of freedom that this molecular architecture provides will contribute to designing next-generation plasmonic photocatalyst with high efficiency and selectivity.

\section{Experimental Section}

Sample/Tip Preparation: The $\mathrm{Au}(111)$ single crystal bead was fabricated by Clavilier's method and was $\mathrm{H}_{2}$ flame-annealed before use.
After annealing, the facets were re-located and directly fixed to a Au substrate. STM measurements were carried out with the bare $\mathrm{Au}(111)$ facet to show that the surface was atomically flat with a few terraces (Figure S10, Supporting Information). layer on glassy carbon electrode. Before the thiol treatment, a few cycles cyclic voltammetry (CV) was run in $0.1 \mathrm{M} \mathrm{H}_{2} \mathrm{SO}_{4}$ to clean the surface, followed by rinsing with Mill-Q water and drying under a $\mathrm{N}_{2}$ atmosphere.

The silver tip was sharpened by electrochemical etching in the etching solution of perchloric acid and ethanol $(1: 4 \mathrm{v} / \mathrm{v})$ under $10 \mathrm{~V}$. The diameter of the apex was near 120-160 nm (Figure S11, Supporting Information). The dielectric constants of silver resulted in a larger polarizability than of gold. Besides, in gap-mode TERS, the substrate could have a strong plasmonic coupling with the tip, resulting in an extra field enhancement and a redshift of the plasmon resonance.

Assembly of Molecules on Au Surfaces: The composition of the thiol mixture should be chosen such that phase segregation into microscopic subdomains was avoided. Phase segregation was facilitated when thiols with different chain lengths were present. The energy difference was minimized by choosing thiol pairs with the same carbon chain length but different tail groups.

For thiol monolayers, the bare $A u(111)$ substrates were immersed for $6 \mathrm{~h}$ in a $10 \times 10^{-3} \mathrm{M}$ ethanolic solution of one of the following compounds: phenylthiophenol (Biph), thiophenol (PhSH), MGITC, or mixed thiols to form an SAM on the surface.

For MB molecules, after modification with thiols (carrying an amino functional group) and rinsing with ethanol, the substrate was immersed into $10 \times 10^{-3} \mathrm{M}$ ethanolic MB-NHS solution for $24 \mathrm{~h}$ to allow the formation of chemical bond between the amino group and the NHS ester.

For NB molecules, a similar method was used, which was reported by Willets et al. ${ }^{[14]}$ After modification by thiols (containing a carboxyl functional group) and rinsing with ethanol, substrates were incubated in freshly prepared $20 \times 10^{-3} \mathrm{M} \mathrm{EDC}$ and $40 \times 10^{-3} \mathrm{M} \mathrm{NHS}$ for $4 \mathrm{~h}$. After profusely rinsing with ethanol and blowing with $\mathrm{N}_{2}$, the substrate was immersed into a $10 \times 10^{-3} \mathrm{M}$ ethanoic NB solution for $24 \mathrm{~h}$ at room temperature to allow the formation of chemical bond between dyes and substrates.
The Au electrode was fabricated by directly evaporating $100 \mathrm{~nm} \mathrm{Au}$ 
To form a surface covered by a lower density of dyes, the substrate was immersed into a mixture of $10 \times 10^{-3} \mathrm{M}$ of a thiol without any functional group, and $20 \times 10^{-6} \mathrm{M}$ of a thiol with a functional group, for $6 \mathrm{~h}$. Undecanethiol $\left(\mathrm{C}_{11} \mathrm{H}_{23} \mathrm{SH}\right) / 11$-amino-1-undecanethiol $\left(\mathrm{NH}_{2} \mathrm{C}_{11} \mathrm{H}_{22} \mathrm{SH}\right) / 12$-mercaptododecanoic acid $\left(\mathrm{SHC}_{11} \mathrm{H}_{22} \mathrm{COOH}\right)$, pentanethiol $\left(\mathrm{C}_{5} \mathrm{H}_{11} \mathrm{SH}\right) / 6$-mercaptohexanoic acid $\left(\mathrm{SHC}_{5} \mathrm{H}_{10} \mathrm{COOH}\right)$, 16-mercaptohexadecanoic acid $\left(\mathrm{SHC}_{15} \mathrm{H}_{30} \mathrm{COOH}\right) / 1$-pentadecanethiol $\left(\mathrm{C}_{15} \mathrm{H}_{31} \mathrm{SH}\right)$, as well as thiophenol/4-mercaptobenzoic acid were used for this purpose. All substrates were carefully rinsed with ethanol to remove physisorbed compounds before carrying out further experiments.

EC-SERS and Electrochemical Measurements: A PGSTAT204 modular Autolab potentiostat (Metrohm, CH) was used to conduct all electrochemical and spectrochemical measurements. For SERS measurements, a droplet was left containing $150 \mathrm{~nm} \mathrm{Ag}$ nanoparticles on gold surface, then it formed coffee rings under vacuum chamber which was an ideal substrate for EC-SERS (Figure S12, Supporting Information). The measurements were operated with home-made EC-Raman cell containing of a large surface area platinum wire as counter electrode and a Calomel (Sat'd $\mathrm{KCl}$ ) reference electrode. The scan rate $5 \mathrm{mV} \mathrm{s}^{-1}$ was used for potential control EC-SERS. The optical instrument was the same with TERS Raman spectrometer (NT-MDT, Russia, NTEGRA Spectra Upright) with $632.8 \mathrm{~nm}$ He-Ne laser as excitation source, an air objective (100 $\times, N A=0.7$, Mitutoyo, Japan), and an electron multiplying chargecoupled device (EMCCD, Newton 971 UVB, Andor, Belfast, UK). During the measurement, $2.3 \mathrm{~mW}$ laser power and $2 \mathrm{~s}$ acquisition time were chosen to obtain significant SERS signals.

The $\mathrm{Au}(111)$ Electrochemical Experiment: CV was done with Pipet cell since the $\mathrm{Au}(111)$ facet was relatively small. The $\mathrm{CV}$ of $\mathrm{Au}(111)$ was compared in $0.1 \mathrm{~mol} \mathrm{~L}^{-1} \mathrm{H}_{2} \mathrm{SO}_{4}$ by this method to literatures to calibrate the potential differences result from the quasi-reference electrode.

Furthermore, to measure the surface coverage, the conventional surface-analytical $\mathrm{Au}(111)$ electrochemistry ${ }^{[37]}$ was also applied to integral the charge quantity (Figure S1, Supporting Information). The surface area was estimated by scanning electron microscope image (Figure S2, Supporting Information), and the surface average was calculated by the equilibrium equation.

TERS Measurements: The TERS instrument was described above with a combined STM module. The instrument was calibrated by a standard neon lamp (Renishaw) and Si before experiment operation. TERS maps were collected in STM feedback (constant current mode) with the sample surface be moved by the piezo sample stage in the $x, y$, and $z$ directions while keeping the relative laser-to-tip position fixed. An exposure time of $5 \mathrm{~s}$ was used for all spectra. The $\mathrm{HeNe}$ laser was set at an incident power of $30 \mu \mathrm{W}$ in order to avoid the photo-bleaching and thermal damaging on the surface of nano-antennas. ${ }^{[17,34]}$ During TERS measurements, the bias voltage was set to $0.3 \mathrm{~V}$ (to prevent bias triggered reaction), ${ }^{[38,39]}$ and the tunneling current was kept at $0.2 \mathrm{nA}$ to avoid scratching the sheet. All TERS images were obtained after overnight measurements to minimize the thermal drift of the system.

Data Analysis and Calculations: Analysis method: All spectra shown in this work had been processed by the following methods: background subtraction, Savitzky-Golay smoothing, cosmic ray removal. For significance analysis for low surface intensity, the signals above 3 standard deviation $(3 \sigma 1)$ of noise level were considered valid-resonant for further statics for each spectrum. Normal distribution was applied for each region and the intensity ( $x$-axis) was rescaled for visualization. All the data were analyzed with Matlab_R2017b software with build-in packages.

Noise floor was determined for NB and MB. A region was chosen with blank Raman peak and close to the target band as noise floor. The mean value of noise floor was taken to further analyze (Mnoise). Since the fluctuation could also result from the instable laser intensity, the standard deviation $\sigma 2$ of Mnoise was calculated corresponding to time. Only the signals above of $3 \sigma 2$ were resulted from redox reaction instead of laser fluctuation. It was assumed that the nonresonance structure/resonance Raman intensity would obey normal distribution separately and the integration of the total probability density function was set to be 1 . The data were analyzed first to point out the typical noise region to fit normal distribution and the corresponding sigmaHat was calculated. The data above $3 \sigma 2$ were assigned as the valid resonance 1 signals to fit another normal distribution, the final data were normalized 2 by the ratio of their highest peak (also the ratio in Figure S4, Supporting 3 Information).

Calculation Methods: Raman spectra of free molecules were calculated with Gaussian 09. The basis sets for $\mathrm{C}, \mathrm{N}, \mathrm{S}, \mathrm{O}$, and $\mathrm{H}$ atoms were $6-31+C *$. Full geometry optimizations and frequency analyses were 6 carried out by using Gaussian 09 package. To understand the vibrational 7 spectra of $\mathrm{MB}$, a scaling factor of 0.978 was used for the vibrational 8 frequencies related to the internal coordinators to the force constant 9 matrix calculated at the B $3 \mathrm{LYP} / 6-31+\mathrm{G} *$ level. This moderate level of theory already helped a lot to understand the appearance of bands characteristic of the reduced form.

The metal slab was consisted of four $(6 \times 6)$ gold $(111)$ atomic layers. The molecules were linked via Au-S bond and their LUMO are plotted accordingly in Figure 4. All the simulations were carried out by keeping the two bottom Au layers fixed at the initial coordinates in order to maintain the bulk behavior of the inner part of the slab. DFT calculation was performed by using the CP2K package, Perdew-Burke-Ernzerhof functional with Grimme D3 correction was used to describe the system. Unrestricted Kohn-Sham DFT had been used as the electronic structure method in the framework of the Gaussian and plane waves method. The Goedecker-Teter-Hutter (GTH) pseudopotentials, DZVP-MOLOPT-GTH basis sets were utilized to describe the molecules. A plane-wave energy cut-off of 500 Ry had been employed. The electronic structure analysis was carried out in terms of the projected density of state (PDOS) of the molecules adsorbed on the $A u(111)$ surface.

\section{Supporting Information}

Supporting Information is available from the Wiley Online Library or 28 from the author.

\section{Acknowledgements}

The authors thank the central HPC clusters of ETH Zurich for supercomputing resources. H.Y. also thanks Sino-Swiss Science and Technology Cooperation for a student fellowship and support through the research. The authors also thank L. Q. Zheng, J. Szczerbinski, and Prof. B. Ren for helpful discussions. This work was supported by the NSFC $(21522508,21775127$ and 21874111) and by the Sino-Swiss Science and Technology Cooperation program (EG22-122016).

\section{Conflict of Interest}

The authors declare no conflict of interest.

\section{Keywords}

dye redox reaction, hot electrons, Raman intensity fluctuation, tip-enhanced Raman spectroscopy (TERS), tunneling mechanism

Received: July 10,2019 50

Revised: August 30, 201951

Published online:

[1] M. L. Brongersma, N. J. Halas, P. Nordlander, Nat. Nanotechnol. 2015, 10, 25.

[2] S. Mubeen, J. Lee, N. Singh, S. Kramer, G. D. Stucky, M. Moskovits, Nat. Nanotechnol. 2013, 8, 247.

[3] S. Linic, P. Christopher, D. B. Ingram, Nat. Mater. 2011, 10, 911. 11 14 15 16 17 18 18 19 20 21 22 22 36 
[4] S. Mukherjee, F. Libisch, N. Large, O. Neumann, L. V. Brown, J. Cheng, J. B. Lassiter; E. A. Carter, P. Nordlander, N. J. Halas, Nano Lett. 2013, 13, 240.

[5] B. de Nijs, F. Benz, S. J. Barrow, D. O. Sigle, R. Chikkaraddy, A. Palma, C. Carnegie, M. Kamp, R. Sundararaman, P. Narang, O. A. Scherman, J. Baumberg, Nat. Commun. 2017, 8, 994.

[6] Y. F. Huang, M. Zhang, L. B. Zhao, J. M. Feng, D. Y. Wu, B. Ren Z. Q. Tian, Angew. Chem., Int. Ed. 2014, 53, 2353.

[7] E. M. van Schrojenstein Lantman, T. Deckert-Gaudig, A. J. Mank, V. Deckert, B. M. Weckhuysen, Nat. Nanotechnol. 2012, 7, 583.

[8] L. B. Zhao, M. Zhang, Y. F. Huang, C. T. Williams, D. Y. Wu, B. Ren, Z. Q. Tian, J. Phys. Chem. Lett. 2014, 5, 1259.

[9] A. da Silva, T. Rodrigues, V. Correia, T. Alves, R. Alves, R. Ando, F. Ornellas, J. Wang, L. Andrade, H. Camargo, Angew. Chem., Int. Ed. 2016, 55, 7111.

[10] E. Cortés, W. Xie, J. Cambiasso, A. S. Jermyn, R. Sundararaman, P. Narang, S. Schlücker, S. A. Maier, Nat. Commun. 2017, 8, 14880.

[11] J. H. Zhong, X. Jin, L. Meng, X. Wang, H. S. Su, Z. L. Yang, C. T. Williams, B. Ren, Nat. Nanotechnol. 2016, 12, 132.

[12] R. Zhang, Y. Zhang, Z. C. Dong, S. Jiang, C. Zhang, L. G. Chen, L. Zhang, Y. Liao, J. Aizpurua, Y. Luo, J. L. Yang, J. G. Hou, Nature 2013, 498, 82 .

[13] D. Kurouski, M. Mattei, R. P. van Duyne, Nano Lett. 2015, 15, 7956.

[14] A. J. Wilson, N. Y. Molina, K. A. Willets, J. Phys. Chem. C 2016, 120, 21091.

[15] C. D. Bain, E. B. Troughton, Y. T. Tao, J. Evall, G. M. Whitesides, R. G. Nuzzo, J. Am. Chem. Soc. 1989, 111, 321.

[16] C. Vericat, M. E. Vela, G. Benitez, P. Carro, R. C. Salvarezza, Chem. Soc. Rev. 2010, 39, 1805.

[17] B. Pettinger, B. Ren, G. Picardi, R. Schuster, G. Ertl, J. Raman Spectrosc. 2005, 36, 541.

[18] X. Qian, S. R. Emory, S. Nie, J. Am. Chem. Soc. 2012, 134, 2000.

[19] a) S. Nie, S. R. Emory, Science 1997, 275, 1102. b) W. Zhang, M. Caldarola, B. Pradhan, M. Orrit, Angew. Chem., Int. Ed. 2017, 56, 3566.

[20] K. Wu, J. Chen, J. R. McBride, T. Lian, Science 2015, 349, 632.

[21] D. P. dos Santos, M. L. A. Temperini, A. G. Brolo, J. Am. Chem. Soc. 2012, 134, 13492 .
[22] B. Foerster, A. Joplin, K. Kaefer, S. Celiksoy, S. Link, C. Sönnichsen, 1 ACS Nano 2017, 11, 2886.

[23] Y. Zhang, S. He, W. Guo, Y. Hu, J. Huang, J. R. Mulcahy, W. D. Wei, Chem. Rev. 2018, 118, 2927.

[24] J. R. M. Saavedra, A. Asenjo-Garcia, F. J. Garcia de Abajo, ACS Photonics 2016, 3, 1637.

[25] a) Y. Kim, A. J. Wilson, P. K. Jain, ACS Catal. 2017, 7, 4360. b) E. Fung, O. Adak, G. Lovat, D. Scarabelli, L. Venkataraman, Nano Lett. 2017, 17, 1255.

[26] C. E. D. Chidsey, Science 1991, 251, 919.

[27] S. H. Choi, B. Kim, C. D. Frisbie, Science 2008, 320, 1482.

[28] M. A. Reed, C. Zhou, M. R. Deshpande, C. J. Muller, T. P. Burgin, L. Jones II, J. M. Tour, Ann. N. Y. Acad. Sci. 1998, 852, 133.

[29] R. P. Gautam, Y. T. Lee, G. L. Herman, C. M. Moreno, E. C. M. Tse, C. J. Barile, Angew. Chem., Int. Ed. 2018, 57, 13480.

[30] A. J. Wilson, K. A. Willets, Analyst 2016, 141, 5144.

[31] a) J. Li, S. K. Cushing, F. Meng, T. R. Senty, A. D. Bristow, N. Wu, Nat. Photonics 2015, 9, 601. b) L. Y. Hsu, W. Ding, G. C. Schatz J. Phys. Chem. Lett. 2017, 8, 2357.

[32] a) M. Banik, P. Z. El-Khoury, A. Nag, A. Rodriguez-Perez, N. Guarrottxena, G. C. Bazan, V. A. Apkarian, ACS Nano 2012, 6, 10343. b) D. A. Nelson, Z. D. Schultz, J. Phys. Chem. C 2019, 123, 20639. c) J. M. Marr, Z. D. Schultz, J. Phys. Chem. Lett. 2013, 4, 3268.

[33] L. Opilik, Ü. Dogan, C. Li, B. Stephanidis, J. Li, R. Zenobi, J. Phys. Chem. C 2016, 120, 20828.

[34] T. E. Tesema, B. Kafle, M. G. Tadesse, T. G. Habteyes, J. Phys. Chem. C 2017, 121, 7421.

[35] J. Szczerbiński, L. Gyr, J. Kaeslin, R. Zenobi, Nano Lett. 2018, 18, 6740.

[36] L. Zhou, F. D. Swearer, C. Zhang, H. Robatjazi, H. Zhao, L. Henderson, L. Dong, P. Christopher, E. Carter, P. Nordlander, N. Halas, Science 2018, 362, 69.

[37] M. J. Esplandiu, H. Hagenström, D. M. Kolb, Langmuir 2001, 17, 31 828.

[38] H. Bi, C. Palma, Y. Gong, P. Hasch, M. Elbing, M. Mayor, J. Reichert, V. J. Barth, J. Am. Chem. Soc. 2018, 140, 4835.

[39] S. Fatayer, B. Schuler, W. Steurer, I. Scivetti, J. Repp, L. Gross, M. Persson, G. Meyer, Nat. Nanotechnol. 2018, 3, 376. 


\section{small

Reprint Order Form 2019

- please return with your proofs -
Editorial Office:

Wiley-VCH Verlag, Boschstrasse 12 69469 Weinheim, Germany

Tel.: (+49) 6201606235

Fax: $(+49) 6201606500$

E-mail: advenergymat@wiley-vch.de http://www.small-journal.com

\section{Manuscript No.}

Please send me and bill me for

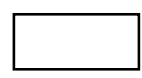
no. of reprints via $\square$ airmail ( +25 Euro) $\square$ surface mail

high-resolution PDF file (330 Euro).

My e-mail address:

Please note: It is not permitted to present the PDF file on the internet or on company homepages

$\star$ Special Offer $\star$ If you order 200 or more reprints you will get a PDF file for half price.

\section{Information regarding VAT}

Please note that from German sales tax point of view, the charge for Reprints, Issues or Posters is considered as "supply of goods" and therefore, in general, such delivery is a subject to German sales tax. However,this regulation has no impact on customers located outside of the European Union. Deliveries to customers outside the Community are automatically tax-exempt. Deliveries within the Community to institutional customers outside of Germany are exempted from the German tax (VAT) only if the customer provides the supplier with his/her VAT number. The VAT number (value added tax identification number) is a tax registration number used in the countries of the European Union to identify corporate entities doing business there. It starts with a country code (e.g. FR for France, GB for Great Britain) and follows by numbers.

\section{Cover Posters}

Posters are available of all the published covers and frontispieces in two sizes

DIN A2 42 x $60 \mathrm{~cm} / 17$ x 24in (one copy: 39 Euro)

DIN A1 $60 \times 84$ cm/ 24 x 33in (one copy: 49 Euro)

Postage for shipping posters overseas by airmail:

\section{+ 25 Euro}

Postage for shipping posters within Europe by surface mail: +15 Euro
Mail reprints / cover posters to:

\section{Invoice address:}

Date, Signature

Stamp

VAT no.:

(institutes / companies in EU countries only)

\section{Purchase Order No.:}

\section{Credit Card Payment}

VISA, MasterCard, AMERICAN EXPRESS

Please use the Credit Card Token Generator located at the website below to create a token for secure payment. The token will be used instead of your credit card number.

\section{Credit Card Token Generator:}

https://www.wiley-vch.de/editorial_production/index.php

Please transfer your token number to the space below.

\section{Credit Card Token Number:}

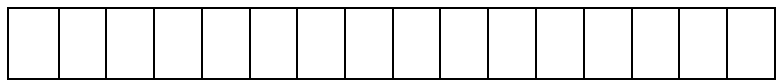

Price list for reprints (The prices include mailing and handling charges. All Wiley-VCH prices are exclusive of VAT)

\begin{tabular}{c|cccccr}
\hline \multirow{2}{*}{ No. of pages } & \multicolumn{5}{c}{ Price (in Euro) for orders of } \\
& 50 copies & 100 copies & 150 copies & 200 copies & 300 copies & 500 copies \\
\hline $1-4$ & 345 & 395 & 425 & 445 & 548 & 752 \\
$5-8$ & 490 & 573 & 608 & 636 & 784 & 1077 \\
$9-12$ & 640 & 739 & 786 & 824 & 1016 & 1396 \\
$13-16$ & 780 & 900 & 958 & 1004 & 1237 & 1701 \\
$17-20$ & 930 & 1070 & 1138 & 1196 & 1489 & 2022 \\
\hline for every additional 4 pages & 147 & 169 & 175 & 188 & 231 & 315 \\
\hline
\end{tabular}

$\star$ Special Offer $\star$ If you order 200 or more reprints you will get a PDF file for half price.

Wiley-VCH Verlag GmbH \& Co. KGaA; Location of the Company: Weinheim;

Chairman of the Supervisory Board: John Kritzmacher,

Trade Register: Mannheim, HRB 432833, General Partner: John Wiley \& Sons GmbH, 